Pacific Journal of Mathematics

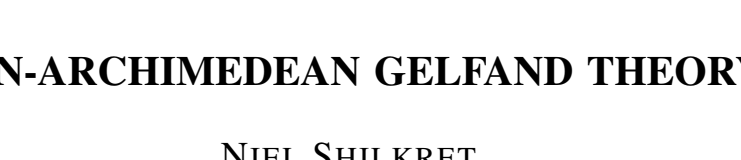




\section{NON-ARCHIMEDEAN GELFAND THEORY}

\section{NEIL SHILKRET}

It is shown that under certain conditions a specified subalgebra $X_{0}$ of a non-Archimedean Banach algebra is isometrically isomorphic to the space of all continuous functions from a compact zero-dimensional Hausdorff space to the ground field; this generalizes a recent result in which $X_{0}$ is assumed to coincide with the entire algebra.

It is well-known that a complex commutative $B^{*}$-algebra with identity is isometrically isomorphic to the space of all continuous functions on the compact Hausdorff space of its maximal ideals with the Gelfand topology. Narici [7] has proved a similar representation theorem for non-Archimedean algebras (see Corollary 6.2). In order that the Gelfand topology be defined as for complex Banach algebras, Narici assumed that the quotient field of the algebra modulo each of its maximal ideals was isomorphic to the ground field. In the present paper an extension of Narici's result is given in which no assumption is made concerning these quotient fields. As preliminaries to this result, a subalgebra of the given algebra, having several pleasant properties, will be introduced, and some natural generalizations of the definition of the Gelfand topology will be considered.

In $\S 1, X$ will denote a commutative algebra with identity $e$ over the field $F ; \mathscr{C}$ will denote the set of maximal ideals of $X ; x(M)$, where $x \in X$ and $M \in \mathscr{C}$, will denote the coset of $M$ to which $x$ belongs; $x(\mathscr{C l})$ will denote the set $\{x(M) \mid M \in \mathscr{C}\}$; and $\mathscr{R}$ will denote the Jacobson radical, $\cap \mathscr{M}$. The field $X / M, M \in \mathscr{M}$, will be considered as an extension field of $F$ (which may or may not be proper) under the identification $\alpha \leftrightarrow \alpha e(M)$ for each $\alpha \in F$. Then $\sigma(x)$, the spectrum of $x \in X$, is equal to $x(\mathscr{C}) \cap F$.

In $\S 2$ and $\S 3$, it will be assumed, moreover, that $F$ is complete with respect to a nontrivial rank one valuation, | |, and $X$ is a nonArchimedean commutative Banach algebra with identity $e$ over $F$; i.e.,

(1) $X$ is a non-Archimedean Banach space satisfying the ultrametric inequality $\|x+y\| \leqq \max (\|x\|,\|y\|)$;

(2) $\|x y\| \leqq\|x\|\|y\|$;

(3) $\|e\|=1$.

1. The Gelfand subalgebra.

DEFINITION. The set

$$
X_{0}=\{x \in X \mid x(M) \in F \quad \text { for all } M \in \mathscr{C}\}
$$


will be called the Gelfand subalgebra of $X$.

Objects related to $X_{0}$ will be subscripted with a zero; e.g., $\mathscr{A}_{0}$ will denote the set of all maximal ideals of $X_{0}$.

Certainly $X_{0}$ is a subalgebra containing the identity.

The set of maximal ideals $M$ in $X$ such that $X / M=F$ will be called $\mathscr{C l}^{\prime}$; the set of maximal ideals $M_{0}$ in $X_{0}$ such that $X_{0} / M_{0}=F$ will be called $\mathscr{L}_{0}^{\prime}$.

THEOREM 1. (a) If $x \in X_{0}$ is invertible in $X$, then $x^{-1} \in X_{0}$.

(b) The function $\Psi: M \rightarrow M_{0}=M \cap X_{0}$ carries maximal ideals of $X$ into maximal ideals of $X_{0}$ having the property $X_{0} / M_{0}=F$; thus, denoting $\Psi(\mathscr{L})$ by $\mathscr{H}_{0}^{\prime \prime}$, we have $\mathscr{L}_{0}^{\prime \prime} \subset \mathscr{H}_{0}^{\prime} \subset \mathscr{H}_{0}$.

(c) If $M \in \mathscr{M}, M_{0}=M \cap X_{0}$ and $x \in X_{0}$, then $x(M)=x\left(M_{0}\right)$.

(d) If $x \in X_{0}$

$$
\sigma(x)=\sigma_{X_{0}}(x)=x(\mathscr{H})=x\left(\mathscr{C}_{0}\right) \cap F=x\left(\mathscr{C}_{0}^{\prime}\right)=x\left(\mathscr{H}_{0}^{\prime \prime}\right) .
$$

(e ) $\mathscr{R}_{0} \subset \mathscr{R} \subset X_{0}$. Hence $\mathscr{R} \oplus F \subset X_{0}$, and $X_{0}$ is semisimple if $X$ is.

(f) If $\sigma(x)=\phi, x \notin X_{0}$.

(g) If $X \supset K \supset F$, where $K$ is a field, then $K \cap X_{0}=F$. Consequently, if $X$ is a field $X_{0}=F$.

(h) $X_{0}=X$ if and only if $X / M=F$ for all $M \in \mathscr{M}(i . e$. , if and only if $\mathscr{L}=\mathscr{C}^{\prime}$ ).

(i) $\quad X_{0}=\bigcap_{M \in \mathscr{M}} M \oplus F$.

Proof. (a) $x^{-1}(M)=x(M)^{-1}$.

(b) Apply the fundamental theorem of homomorphisms to the mapping

$$
\begin{aligned}
& X_{0} \longrightarrow X / M \\
& x \longrightarrow x(M) .
\end{aligned}
$$

(c) If $x \in X_{0}$ and $\alpha \in F$,

$$
\begin{aligned}
x(M)=\alpha & \Longleftrightarrow x-\alpha \in M \\
& \Longleftrightarrow\left(\text { since } x, \alpha \in X_{0}\right) x-\alpha \in M_{0} \\
& \Longleftrightarrow x\left(M_{0}\right)=\alpha .
\end{aligned}
$$

(d) From (c) it follows that for $x \in X_{0}$,

$$
\sigma(x)=x(\mathscr{C})=x\left(\mathscr{C}_{0}^{\prime \prime}\right) \subset x\left(\mathscr{C}_{0}^{\prime}\right) \subset x\left(\mathscr{C}_{0}\right) \cap F=\sigma_{X_{0}}(x) .
$$

However, (a) implies $\sigma(x)=\sigma_{X_{0}}(x)$.

(e) These statements are all easy. 
(f) If $x \in X_{0}, \sigma(x)=x(\mathscr{C})$, which cannot be empty.

(g) This follows from (f), since each $x \in K-F$ has empty spectrum.

(h) and (i) are clear.

Parts $(\mathrm{g})$ and $(\mathrm{h})$ show the extreme cases $X_{0}=F$ and $X_{0}=X$ are possible.

Lemma 2.1. Let $M$ be a maximal ideal of $X$, and let $x$ be an algebraic element of $X$ having minimal polynomial $m(t)=\prod p_{i}(t)^{k_{i}}$, where the polynomials $p_{i}(t)$ are irreducible over $F$. Then $x(M)(\in X / M)$ $i s$ algebraic over $F$, and the irreducible polynomial over $F$ which $x(M)$ satisfies is one of the $p_{i}$. Conversely, for each of the irreducible factors $p_{i}$ of $m$, there is a maximal ideal $M_{i}$ such that $x\left(M_{i}\right)\left(\in X / M_{i}\right)$ satisfies $p_{i}$.

Proof. Since $m(x)=0, m(x(M))=\Pi p_{i}(x(M))^{k_{i}}=0$, for any maximal ideal $M$, and, indeed, one of the $p_{i}$ is the irreducible polynomial which $x(M)$ satisfies.

Each of the elements $p_{i}(x)$ is a divisor of zero, so that each of these elements is a nonunit of $X$ and is contained in some maximal ideal $M_{i}$. Therefore $p_{i}\left(x\left(M_{i}\right)\right)=0$.

From this one readily obtains

THeOREm 2. An algebraic element $x \in X$ is in $X_{0}$ if and only if its minimal polynomial factors into a product of linear polynomials.

COROLlary 2.1. If $F$ is algebraically closed, $X_{0}$ contains all algebraic elements of $X$.

Corollary 2.2. (a) $X_{0}$ and $\left(X_{0}\right)_{0}$ have the same algebraic elements.

(b) If $X$ is algebraic (i.e., all elements of $X$ are algebraic), $\left(X_{0}\right)_{0}=X_{0}$.

(c) If $X$ is finite dimensional, $\left(X_{0}\right)_{0}=X_{0}$.

Corollary 2.2 suggests the question is $\left(X_{0}\right)_{0}=X_{0}$ for every algebra $X$ ? An answer to this question has not been found.

ExAMPLE. Let $X$ be a commutative Banach algebra with identity over the real numbers, $R$. Then $X_{0}$ is complete by the completeness of $R$ (and the fact $\left\{x_{n}\right\}$ being Cauchy implies $\left\{\left(x_{n}(M)\right\}\right.$ is Cauchy for each $M \in \mathscr{L})$. Thus, for each $M_{0} \in \mathscr{H}_{0}, X_{0} / M_{0}$ is a commutative Banach algebra which is a field. Hence by the Mazur-Gelfand theorem, 
$X_{0} / M_{0}$ is isomorphic to either the real numbers or the complex numbers, $C$. But according to the above, $X_{0} / M_{0}=C$ is impossible. Therefore $X_{0} / M_{0}=R$ for each $M_{0} \in \mathscr{M}_{0}$ or, equivalently, $\left(X_{0}\right)_{0}=X_{0}$.

\section{Spaces of maximal ideals.}

Definition. The weakest topology on $\mathscr{L}^{\prime}$ for which each of the functions

$$
\begin{aligned}
\hat{x}: \mathscr{C}^{\prime} & \longrightarrow F \\
M & \longrightarrow x(M)
\end{aligned}
$$

is continuous will be called the Gelfand topology. This definition may be applied to the algebra $X_{0}$ to obtain a Gelfand topology on $\mathscr{M}_{0}^{\prime}$.

If $X_{0}=X$, then $\mathscr{C}_{0}^{\prime}=\mathscr{C}^{\prime}=\mathscr{C}$ and the above definitions reduce to the usual definition of the Gelfand topology.

These topological spaces will be regarded as uniform spaces with the uniformity which has a base consisting of sets of the form

$$
U=\left\{\left(M, M^{\prime}\right)|\quad| x_{i}(M)-x_{i}\left(M^{\prime}\right) \mid<\varepsilon, i=1, \cdots, n\right\} .
$$

As usual, the set $U[M]$ will be written as $V\left(M ; x_{1}, \cdots, x_{n} ; \varepsilon\right)$.

Naturally all statements concerning $\mathscr{K}^{\prime}$ have analogues concerning $\mathscr{H}_{0}^{\prime}$.

Theorem 3. $\mathscr{M}^{\prime}$ is complete in the Gelfand topology.

Proof. Let $\left\{M_{a}\right\}$ be a Cauchy net in $\mathscr{C}^{\prime}$. We show that $M=$ $\left\{x \in X \mid x\left(M_{a}\right) \rightarrow 0\right\}$ is a maximal ideal in $\mathscr{C}^{\prime}$ and that $M_{a} \rightarrow M$.

A modification of the usual proof shows that the spectral radius, $r_{\sigma}(x)=\sup _{\alpha \in \sigma(x)}|\alpha|$ satisfies

$$
r_{o}(x) \leqq\|x\|
$$

Thus if $x \in M$ and $y \in X,\left|y\left(M_{a}\right) x\left(M_{a}\right)\right| \leqq\|y\|\left|x\left(M_{a}\right)\right| \rightarrow 0$, from which it readily follows that $M$ is an ideal. The mapping

$$
\begin{aligned}
& F \longrightarrow X / M \\
& \alpha \longrightarrow \alpha e+M
\end{aligned}
$$

is an isomorphism, because $\alpha e \in M$ implies $\alpha=\lim _{a} \alpha e\left(M_{a}\right)=0$. But, since $\left\{M_{a}\right\}$ is a Cauchy net, the $M_{a}$ are eventually close of order $U=$ $\left\{\left(M, M^{\prime}\right)|| x(M)-x\left(M^{\prime}\right) \mid<\varepsilon\right\}$. Therefore, for each $x$ there is an $\alpha \in F$ such that 


$$
\begin{array}{r}
x\left(M_{a}\right) \longrightarrow \alpha, \\
(x-\alpha e)\left(M_{a}\right) \longrightarrow 0, \\
x+M=\alpha e+M ;
\end{array}
$$

which implies the isomorphism $\alpha \rightarrow \alpha e+M$ is onto. Hence $M \in \mathscr{C}^{\prime}$.

For a point $x \in X$, let $\alpha=\lim x\left(M_{a}\right)$. Then $\lim (x-\alpha e)\left(M_{a}\right)=0$, $x-\alpha e \in M$ and $x(M)=\alpha e(M)=\alpha=\lim x\left(M_{a}\right)$. Thus $M_{a} \in V(M ; x ; \varepsilon)$ for $a$ sufficiently large. Hence $M_{a} \rightarrow M$, since neighborhoods of this type constitute a subbase.

An immediate consequence of Theorem 3 is that $\mathscr{M}^{\prime}$ is compact if and only if it is totally bounded. In connection with the compactness of $\mathscr{C l}^{\prime}$, note that a modification of the proof for complex Banach algebras shows that if $F$ is locally compact, $\mathscr{C}^{\prime}$ is compact.

THEOREM 4. The Gelfand topology on $\mathscr{M}^{\prime}$ is 0-dimensional, totally disconnected and Hausdorff.

Proof. It follows from the ultrametric inequality which the valuation satisfies that the collection of sets of the form $V\left(M ; x_{1}, \cdots, x_{n} ; \varepsilon\right)$ forms a base consisting of sets which are simultaneously open and closed. Hence $\mathscr{A l}^{\prime}$ is 0-dimensional.

Once it is shown that $\mathscr{C}^{\prime}$ is Hausdorff, it will follow that $\mathscr{M}^{\prime}$ is totally disconnected. For if $A$ is a component of a 0 -dimensional Hausdorff space, and it is assumed there are two distinct points $x$, $y \in A$, then an open and closed neighborhood of $x$ not containing $y$ contradicts the connectedness of $A$. But $\mathscr{C}^{\prime}$ is Hausdorff because the family $\{\hat{x}\}$ separates points.

We state below three results that are simple extensions of results due to Beckenstein [2]:

The topology on $\mathscr{C l}^{\prime}$ induced by the hull-kernel topology on $\mathscr{C}$ is weaker than the Gelfand topology on 'L''.

The family $\{\hat{x}\}, x \in X$, is regular with respect to the Gelfand topology (i.e., it separates point and closed sets of the Gelfand topology) if and only if the hull-kernel topology and the Gelfand topology coincide.

If $\mathscr{C}^{\prime}$ is compact in the Gelfand topology, $\{\hat{x}\}, x \in X$, is regular if and only if $\mathscr{C}^{\prime}$ is Hausdorff in the hull-kernel topology.

One might topologize $\mathscr{C}$ with the functions $\{\hat{x}\}, x \in X_{0}$; however, $\mathscr{M}$ topologized in this fashion need not enjoy the same properties that $\mathscr{C}^{\prime}$ does. For example, $\mathscr{C}$ may receive the trivial topology 
(this happens if and only if $X_{0}=\mathscr{R} \oplus F$ ).

3. Representation theorems. For a closed subspace $A$ of a normed linear space $Y$, the symbol \|\| on $Y / A$ will denote the infimum norm $\|y+A\|=\inf _{a \in A}\|y+a\|$. Then the identification of $\alpha \in F$ with $\alpha e(M) \in(X / M,\|\|), M \in \mathscr{C}$, is an isometry as well as an isomorphism.

We define the rings $V=\{x \in X \mid\|x\| \leqq 1\}$ and

$$
W=\{x \in X \mid\|x(M)\| \leqq 1 \text { for all } M \in \mathscr{C}\} \text {. }
$$

Certainly $V \subset W$.

For $T$ a compact topological space we denote by $F(T)$ the Banach algebra of all continuous $F$-valued functions on $T$ with pointwise operations and $\|f\|=\max _{t \in T}|f(t)|$.

Note that $X_{0}$ is a closed subalgebra since $F$ is complete.

In this section $\mathscr{C l}_{0}^{\prime}$ (which coincides with $\mathscr{H}$ if $X_{0}=X$ ) will be assumed to have the Gelfand topology.

Lemma 5.1. If $\left(X_{0}\right)_{0}=X_{0}, V_{0}=W_{0}$ and $\mathscr{C}_{0}^{\prime}$ is compact, then $r_{o}(x)=\|x\|$ for each $x \in X$.

Proof. Since $\left(X_{0}\right)_{0}=X_{0}, W_{0}=\left\{x \in X_{0} \mid r_{o}(x) \leqq 1\right\}$. Suppose $x \in X_{0}$ is such that

$$
r_{\sigma}(x)<\|x\| \text {. }
$$

Since $\left|x\left(\mathscr{L}_{0}^{\prime}\right)\right|$ is a continuous image of a compact set, there exists $\alpha \in x\left(\mathscr{H}_{0}^{\prime}\right)$ such that $|\alpha|=\sup \left|x\left(\mathscr{C}_{0}^{\prime}\right)\right|=r_{o}(x)$. Then

$$
1=r_{o}(x / \alpha)<\|x / \alpha\|,
$$

which implies $x / \alpha \in W_{0}$ and $x / \alpha \notin V_{0}$, contradicting the hypothesis.

Theorem 5. If $\left(X_{0}\right)_{0}=X_{0}, V_{0}=W_{0}$ and $\mathscr{C}_{0}^{\prime}$ is compact, then $X_{0}$ is isometrically isomorphic to $F\left(\mathscr{L}_{0}^{\prime}\right)$.

Proof. Consider

$$
\begin{aligned}
h: X_{0} & \longrightarrow F\left(\mathscr{C}_{0}^{\prime}\right) \\
x & \longrightarrow \widehat{x} .
\end{aligned}
$$

This is clearly a homomorphism. But

$$
\|\hat{x}\|=\max _{M \in \mathscr{M}_{0}^{\prime}}|\hat{x}(M)|=r_{\sigma}(x)=\|x\|,
$$

so that $X_{0}$ is isometrically isomorphic to $h\left(X_{0}\right)$. But $h\left(X_{0}\right)$ separates 
points, contains constants and, by virtue of the map $h$, is complete. The theorem now follows from a result of Kaplansky [5] (see also Cantor [3] and Chernoff et al. [4]): If $B$ is a subalgebra of $F(T)$, where $T$ is a compact, totally disconnected topological space, and $B$ separates points and contains constants, then $B$ is dense in $F(T)$.

Lemma 6.1. If $V=W$ and $\mathscr{C}_{0}^{\prime}$ is compact, then $r_{o}(x)=\|x\|$ for each $x \in X_{0}$.

Proof. As in Lemma 5.1, $r_{\sigma}(x)<\|x\|$ would lead to $x / \alpha \in W \cap X_{0}=$ $\left\{x \in X_{0} \mid r_{\sigma}(x) \leqq 1\right\}$ and $x / \alpha \notin V \cap X_{0}$.

THEOREM 6. If $V=W$ and $\mathscr{C l}_{0}^{\prime}$ is compact, then $X_{0}$ is isometrically isomorphic to $F\left(\mathscr{C l}_{0}^{\prime}\right)$.

Proof. Choose $h$ as above.

Corollary 6.1. If $V=W$ and $\mathscr{C l}_{0}^{\prime}$ is compact, then $\left(X_{0}\right)_{0}=X_{0}$.

Proof. By Theorems 4 and 6 we may assume $X=F(T)$, where $T$ is a 0 -dimensional compact Hausdorff space. The corollary is a consequence of the following (Narici [7], Ths. 2 and 3): The maximal ideals of $F(T)$ are the sets of the form $M_{t}=\{f \in F(T) \mid f(t)=0\}$, and $F(T) / M_{t}$ is isomorphic to $F$.

CoRollary 6.2. (Narici) Suppose that $X / M=F$ for every maximal ideal $M$, that $V=W$, and that $\mathscr{C l}$ is compact in the Gelfand topology. Then $X$ is isometrically isomorphic to $F(\mathscr{C l})$ under the mapping $x \rightarrow \widehat{x}$.

Note that $V=W$ implies $V_{0}=W_{0}$. For certainly $V_{0}=V \cap X_{0}$; if $x \in W_{0}$, then

$$
\sup _{M \in \mathscr{M}}\|x(M)\|=\sup _{M \in \mathscr{M}_{0}^{\prime \prime}}\|x(M)\| \leqq \sup _{M \in \mathscr{M}_{0}}\|x(M)\| \leqq 1,
$$

which implies $x \in W$. Hence $W_{0} \subset W \cap X_{0}=V \cap X_{0}=V_{0}$.

Let $\hat{X}$ be a commutative Banach algebra without identity (over a complete nontrivially valued field), and suppose that $X$ is the adjunction of identity to $\hat{X}: X$ is the orthogonal sum of $F$ and $\hat{X}$ (see Monna and Springer [6]), and multiplication in $X$ is defined by $(\alpha+x)(\beta+y)=$ $\alpha \beta+\alpha y+\beta x+x y$. Let $\hat{V}=\{x \in \hat{X} \mid\|x\| \leqq 1\}$. Then $V=\mathfrak{p}+\hat{V}$, where $\mathfrak{D}$ is the ring of integers of $F$. $\mathscr{\mathscr { C }}$ will denote the set of all regular maximal ideals of $\hat{X}$, and $x(\hat{M})$ will denote $x+\hat{M}$, where $\hat{M} \in \hat{\mathscr{C}}$. For each $\hat{M} \in \hat{\mathscr{C}}, \hat{X} / \hat{M}$ contains the field $F e_{\widehat{M}}$, where $e_{\widehat{M}}$ is the identity 
of $\hat{X} / \widehat{M} . F e_{\widehat{M}}$ is isomorphic to $F$ under $\alpha \leftrightarrow \alpha e_{\widehat{M}}$, but $\left\|\alpha e_{\widehat{M}}\right\|=|\alpha|\left\|e_{\widehat{M}}\right\|$, so that this is not an isometry unless $\left\|e_{\widehat{M}}\right\|=1$. Let $\hat{\mathscr{C}}^{\prime}$ denote the set of regular maximal ideals of $\hat{X}$ whose quotient fields are (algebra) isomorphic to $F$. The mapping $M \rightarrow M \cap \hat{X}$ is a 1-to-1 correspondence between $\mathscr{C}-\{\hat{X}\}$ and $\hat{\mathscr{C}}$ which preserves quotient fields (and hence is also a 1-to-1 correspondence between $\mathscr{L}^{\prime}-\{\hat{X}\}$ and $\left.\mathscr{\mathscr { L }}^{\prime}\right)$.

Definition. $\quad \hat{X}_{0}=\left\{x \in \hat{X} \mid x(\hat{M}) \in F e_{\widehat{M}}\right.$ for all $\left.\hat{M} \in \hat{\mathscr{C}}\right\}$.

$$
\hat{W}=\left\{x \in \hat{X} \mid\|x(\hat{M})\| \leqq\left\|e_{\widehat{M}}\right\| \text { for all } \hat{M} \in \hat{\mathscr{C}}\right\} \text {. }
$$

Clearly $F+\hat{X}_{0} \subset X$ is the adjunction of identity to $\hat{X}_{0}$.

LEMMA. (a ) $X_{0}=F \oplus \hat{X}_{0}$. and $\hat{\mathscr{C}}_{0}^{\prime}$.

(b) $M \rightarrow M \cap \hat{X}_{0}$ is a 1-to-1 correspondence between $\mathscr{C}_{0}^{\prime}-\left\{\hat{X}_{0}\right\}$

$$
\text { (c) If } \hat{X} / \hat{M}=F \text { for each } \hat{M} \in \hat{\mathscr{C}} \text {, then } W=\mathfrak{o}+\hat{W} \text {. }
$$

Proof. (a) Since $X / \hat{X}=F, \alpha+x \in X_{0}$ if and only if $(\alpha+x)$ $(M) \in F$ for all $M \in \mathscr{C}-\{\hat{X}\}$. Now suppose $M \in \mathscr{M}-\{\hat{X}\}, \hat{M}=$ $M \cap \hat{X}$ and $u \in \hat{X}$ is an identity modulo $\hat{M}$ (i.e., $\left.u(\hat{M})=e_{\widehat{M}}\right)$. Then $M=\{\alpha+x \in X \mid(\alpha+x) u \in \hat{M}\}$, which implies

$$
((1-u) u)(\hat{M})=e_{\widehat{M}}-e_{\widehat{M}}^{2}=0 \text { and } 1-u \in M .
$$

Therefore, if $\beta \in F$,

$$
\begin{aligned}
x(M)=\beta & \Longleftrightarrow 0 \equiv x-\beta \equiv x-\beta u \quad(M) \\
& \Longleftrightarrow(\text { since } x, \beta u \in \hat{X}) x \equiv \beta u \\
& \Longleftrightarrow x(\hat{M})=\beta e_{\widehat{M}} .
\end{aligned}
$$

Thus by the 1-to-1 correspondence between $\mathscr{C}-\{\hat{X}\}$ and $\hat{\mathscr{C}}$, $\alpha+x \in X_{0}$ if and only if $x \in \hat{X}_{0}$.

(b) follows from (a).

(c) The assumption $\hat{X}=\hat{X}_{0}$ implies $X=X_{0}$, so that $\|(\alpha+x)(M)\|$, $M \in \mathscr{L}$, may be written $|(\alpha+x)(M)|$. Therefore

$$
\begin{aligned}
\alpha+x \in W & \Longleftrightarrow|(\alpha+x)(M)|=|\alpha+x(M)| \leqq 1 \text { for all } M \in \mathscr{M} \\
& \Longleftrightarrow \alpha \in \mathfrak{0} \text { and } x(M) \in \mathfrak{D} \text { for all } M \in \mathscr{C}-\{\hat{X}\} \\
& \Longleftrightarrow \alpha \in \mathfrak{D} \text { and } x(\hat{M}) \in \mathfrak{D} e_{\hat{M}} \text { for all } \hat{M} \in \mathscr{\mathscr { C }} \\
& \Longleftrightarrow \alpha+x \in \mathfrak{D}+\hat{W} .
\end{aligned}
$$

Corollary 6.3. If $\hat{X}_{0}=\hat{X}, \hat{V}=\hat{W}$ and $\mathscr{C l}$ is compact, then $\hat{X}$ is isometrically isomorphic to the subalgebra of functions in $F(\mathscr{C})$ which vanish at $\hat{X}$. 
Proof. If $\hat{X}=\hat{X}_{0}$, then $W=\mathfrak{v}+\hat{W}=\mathfrak{v}+\hat{V}=V$ and $X=X_{0}$, so Corollary 6.2 may be used.

CoRollary 5.1. If $\left(\hat{X}_{0}\right)_{0}=\hat{X}_{0}, \hat{V}_{0}=\hat{W}_{0}$ and $\mathscr{N}_{0}^{\prime}$ is compact, then $\hat{X}_{0}$ is isometrically isomorphic to the subalgebra of functions in $F\left(\mathscr{L}_{0}^{\prime}\right)$ which vanish at $\hat{X}_{0}$.

Recall that if the ground field is locally compact, $\mathscr{C}$ in Corollary 6.3 and $\mathscr{C}_{0}^{\prime}$ in Corollary 5.1 are compact.

\section{Miscellaneous.}

THEOREM 7. Let $Y$ be a commutative normed algebra with identity $e$ over the valued field $F$, and let $X$ be a finite dimensional algebra over $Y$ with $Y$-basis $\left\{u_{1}, \cdots, u_{n}\right\}$ and structure constants defined by $u_{i} u_{j}=\sum_{k} c_{i j}^{k} u_{k}$. If $X$ has an identity, we assume that it coincides with the identity of $Y$ and that $u_{1}=e$. Then, viewing $X$ as a normed linear space with the norm $\left\|\sum a_{i} u_{i}\right\|=\max \left\|a_{i}\right\|, a_{i} \in Y$, the following are equivalent:

(a) $X$ is a normed algebra over $F$.

(b) $\left\|u_{i} u_{j}\right\| \leqq\left\|u_{i}\right\|\left\|u_{j}\right\|$ for all $i$ and $j$.

(c) $c_{i j}^{k} \in V_{Y}$ for all $i, j$ and $k$, where $V_{Y}=\{y \in Y \mid\|y\| \leqq 1\}$.

Proof. The equivalence of (b) and (c) follows from the equalities $\left\|u_{i}\right\|\left\|u_{j}\right\|=\|e\|\|e\|=1$ (which follows from the representations $u_{i}=$ $e u_{i}, u_{j}=e u_{j}$ ) and $\left\|u_{i} u_{j}\right\|=\left\|\sum_{k} c_{i j}^{k} u_{k}\right\|=\max _{k}\left\|c_{i j}^{k}\right\|$.

Certainly (a) implies (b), so that to complete the proof it suffices to show (c) implies (a). It is easy to verify that, independent of the nature of the $c_{i j}^{k}, X$ is an algebra over $F$ and a normed vector space. In fact $X$ is isomorphic as a normed space to an orthogonal direct sum of $n$ copies of $Y$ (which shows that $X$ is complete if $Y$ is). If $e$ is the identity of $X$, our assumption $u_{1}=e$ guarantees that $\|e\|=1$. Therefore, to show (c) implies (a), it only remains to show $\|x y\| \leqq$ $\|x\|\|y\|$ for all $x, y \in X$.

Let $x=\sum a_{i} u_{i}$ and let $y=\sum b_{i} u_{i}$. Then

$$
\begin{aligned}
\|x y\| & =\left\|\sum_{k}\left(\sum_{i, j} a_{i} b_{j} c_{i j}^{k}\right) u_{k}\right\| \\
& \leqq \max _{k}\left(\max _{i, j}\left\|a_{i} b_{j} c_{i j}^{k}\right\|\right) \\
& \leqq \max _{i, j, k}\left\|a_{i}\right\|\left\|b_{j}\right\|\left\|c_{i j}^{k}\right\| \\
& \leqq\|x\|\|y\| .
\end{aligned}
$$

Corollary 7.1. Let $Y$ be as above, and let $X=Y[z]$, where $z$ 
satisfies a monic polynomial, say of degree $n$, with coefficients in $V_{Y}$ (i.e., $z$ is integral over $V_{Y}$ ). Then

(a) $X$ is a normed algebra with $\left\|\sum_{0}^{n-1} y_{i} z^{i}\right\|=\max \left\|y_{i}\right\|$;

(b) $\|z\|=1$, i.e., a unit vector has been adjoined to $Y$;

(c) $\|X\|=\|Y\|$; and

(d) $X$ is a commutative Banach algebra with identity if $Y$ is.

Proof. Choosing $u_{i}=z^{i}, i=0, \cdots, n-1$, a simple induction argument shows that $\left\|z^{k}\right\| \leqq 1$ for all $k$, so that

$$
\left\|u_{i} u_{j}\right\|=\left\|z^{i+j}\right\| \leqq 1=\left\|u_{i}\right\|\left\|u_{j}\right\| \text {. }
$$

This establishes (a); (b) and (c) are obvious; and (d) was established in the proof of the main theorem.

Using Corollary 7.1 it is easy to construct a variety of Banach algebras $X$ in which $F \varsubsetneqq X_{0} \varsubsetneqq X$. For example, let $F$ be a $p$-adic number field not containing $i=\sqrt{-1}$ (see Bachman [1]), let $X=$ $F(i)[x]=F[i][x]$, where $x$ has minimal polynomial $(t-1)^{2}$, and norm $F[i][x]$ in two stages according to Corollary 7.1.

\section{REFERENCES}

1. G. Bachman, Introduction to p-adic numbers and valuation theory, Academic Press, New York, 1964.

2. E. Beckenstein, On regular nonarchimedian Banach algebras, Arch. Math. (Basel) 19 (1968), 424-427.

3. D. G. Cantor, On the Stone-Weierstrass approximation theorem for valued fields, Pacific J. Math. 21 (1967), 473-478.

4. P. R. Chernoff, R. A. Rasala, and W. C. Waterhouse, The Stone-Weierstrass theorem for valuable fields, Pacific J. Math. 27 (1968), 233-240.

5. I. Kaplansky, The Weierstrass theorem in fields with valuation, Proc. Amer. Math. Soc. 1 (1950), 356-357.

6. A. F. Monna and T. A. Springer, Sur la structures des espaces de Banach nonArchimediéns, Indag. Math. 27 (1965), 602-614.

7. L. Narici, Nonarchimedian Banach spaces and algebras, Arch. Math. (Basel) 19 (1968), 428-435.

8. M. Van der Put, Algèbras de fonctions continues p-adiques, Indag. Math. 30 (1968), 401-420 (from the author's dissertation, University of Utrecht, 1967).

Received June 4, 1969. Taken from the dissertation submitted to the Faculty of the Polytechnic Institute of Brooklyn in partial fulfillment of the requirements for the degree of Doctor of Philosophy, 1968, under the guidance of Professor George Bachman.

Polytechnic Institute of BROOKLyn 


\section{PACIFIC JOURNAL OF MATHEMATICS}

\section{EDITORS}

\author{
H. SAMELSON \\ Stanford University \\ Stanford, California 94305 \\ RICHARD PIERCE \\ University of Washington \\ Seattle, Washington 98105
}

J. DugundJI

Department of Mathematics University of Southern California Los Angeles, California 90007

BASIL GORDON*

University of California

Los Angeles, California 90024

\section{ASSOCIATE EDITORS}
E. F. BECKENBACH
B. H. NEUMANN
F. WOLE
K. YOSHIDA

\section{SUPPORTING INSTITUTIONS}

\author{
UNIVERSITY OF BRITISH COLUMBIA \\ CALIFORNIA INSTITUTE OF TECHNOLOGY \\ UNIVERSITY OF CALIFORNIA \\ MONTANA STATE UNIVERSITY \\ UNIVERSITY OF NEVADA \\ NEW MEXICO STATE UNIVERSITY \\ OREGON STATE UNIVERSITY \\ UNIVERSITY OF OREGON \\ OSAKA UNIVERSITY \\ UNIVERSITY OF SOUTHERN CALIFORNIA
}

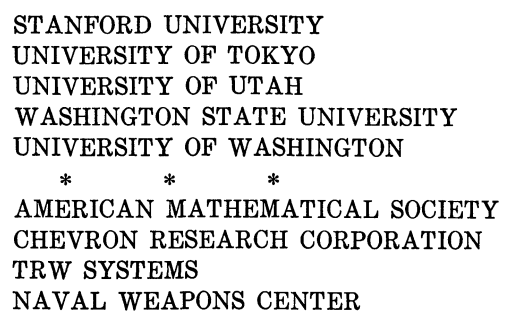

The Supporting Institutions listed above contribute to the cost of publication of this Journal, but they are not owners or publishers and have no responsibility for its content or policies.

Mathematical papers intended for publication in the Pacific Journal of Mathematics should be in typed form or offset-reproduced, (not dittoed), double spaced with large margins. Underline Greek letters in red, German in green, and script in blue. The first paragraph or two must be capable of being used separately as a synopsis of the entire paper. The editorial "we" must not be used in the synopsis, and items of the bibliography should not be cited there unless absolutely necessary, in which case they must be identified by author and Journal, rather than by item number. Manuscripts, in duplicate if possible, may be sent to any one of the four editors. Please classify according to the scheme of Math. Rev. 36, 1539-1546. All other communications to the editors should be addressed to the managing editor, Richard Arens, University of California, Los Angeles, California, 90024.

50 reprints are provided free for each article; additional copies may be obtained at cost in multiples of 50 .

The Pacific Journal of Mathematics is published monthly. Effective with Volume 16 the price per volume (3 numbers) is $\$ 8.00$; single issues, $\$ 3.00$. Special price for current issues to individual faculty members of supporting institutions and to individual members of the American Mathematical Society: $\$ 4.00$ per volume; single issues $\$ 1.50$. Back numbers are available.

Subscriptions, orders for back numbers, and changes of address should be sent to Pacific Journal of Mathematics, 103 Highland Boulevard, Berkeley, California, 94708.

PUBLISHED BY PACIFIC JOURNAL OF MATHEMATICS, A NON-PROFIT CORPORATION

Printed at Kokusai Bunken Insatsusha (International Academic Printing Co., Ltd.), 7-17, Fujimi 2-chome, Chiyoda-ku, Tokyo, Japan.

* Acting Managing Editor. 


\section{Pacific Journal of Mathematics}

\section{Vol. 32, No. $2 \quad$ February, 1970}

Harry P. Allen and Joseph Cooley Ferrar, Jordan algebras and exceptional subalgebras of the exceptional algebra $E_{6} \ldots \ldots \ldots \ldots \ldots \ldots \ldots 283$

David Wilmot Barnette and Branko Grünbaum, Preassigning the shape of a

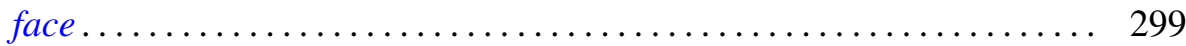

Robert Francis Craggs, Involutions of the 3-sphere which fix 2-spheres . . . . 307

David William Dean, Bor-Luh Lin and Ivan Singer, On k-shrinking and $k$-boundedly complete bases in Banach spaces ................ 323

Martin Engert, Finite dimensional translation invariant subspaces ....... 333

Kenneth Lewis Fields, On the global dimension of residue rings ......... 345

Howard Gorman, The Brandt condition and invertibility of modules ....... 351

Benjamin Rigler Halpern, A characterization of the circle and interval ..... 373

Albert Emerson Hurd, A uniqueness theorem for second order quasilinear

hyperbolic equations ............................... 415

James Frederick Hurley, Composition series in Chevalley algebras ...... 429

Meira Lavie, Disconjugacy of linear differential equations in the complex

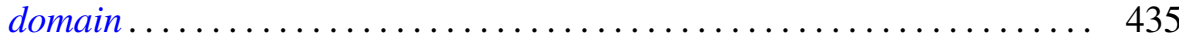

Jimmie Don Lawson, Lattices with no interval homomorphisms ......... 459

Roger McCann, A classification of center-foci ................. 467

Evelyn Rupard McMillan, On continuity conditions for functions . . . . . . . 479

Graciano de Oliveira, A conjecture and some problems on permanents .... 495

David L. Parrott and S. K. Wong, On the Higman-Sims simple group of order $44,352,000$.

Jerome L. Paul, Extending homeomorphisms ................. 517

Thomas Benny Rushing, Unknotting unions of cells .............. 521

Peter Russell, Forms of the affine line and its additive group.......... 527

Niel Shilkret, Non-Archimedean Gelfand theory ................. 541

Alfred Esperanza Tong, Diagonal submatrices of matrix maps.......... 551 